\title{
Supra-annular aortic valve replacement: technique and early outcomes
}

\author{
Nathanael Shraer ${ }^{1}$, Ramzi Ramadan ${ }^{1}$, Alexandre Azmoun ${ }^{1}$, and Julien Guihaire ${ }^{1}$ \\ ${ }^{1}$ Hospital Marie Lannelongue
}

March 20, 2021

\begin{abstract}
OBJECTIVES: The supra-annular aortic valve replacement (SA-AVR) allows for implantation of larger prostheses. We describe the technique, early post-operative outcomes and hospital mortality. METHODS: Patients who underwent SA-AVR with the Carpentier-Edwards Magna Ease bioprosthesis between December 2010 and December 2017 were retrospectively reviewed. The prosthesis was sutured to the aortic annulus along the coronary sinuses, and in a supra-annular position along the noncoronary sinus. RESULTS: 115 patients were included (mean age: 71,6 years $\pm 9,4$ ). Mean bioprosthesis diameter was $23,3 \pm$ 1,7 mm. Four early deaths were observed in the ICU. Early post-operative complications included: re-operation for bleeding $(\mathrm{n}=6)$, tamponade $(\mathrm{n}=7)$, permanent atrioventricular block $(\mathrm{n}=4)$ and hemodialysis $(\mathrm{n}=2)$. In-hospital mortality was $3,48 \%$. Postoperative echocardiography showed a marked decrease in the mean left ventricle - aorta gradient (50,4 $\pm 16,1 \mathrm{mmHg}$ vs $11,3 \pm 4,05 \mathrm{mmHg}$ ). CONCLUSION: SA-AVR is safe and associated with favourable immediate outcomes in patients with small aortic annulus.
\end{abstract}

TITLE: Supra-annular aortic valve replacement: technique and early outcomes

RUNNING TITLE: Supra-annular aortic valve replacement

AUTHORS: Nathanael Shraer, MD ${ }^{1,2}$, Ramzi Ramadan, MD ${ }^{1}$, Alexandre Azmoun, MD $^{1,2}$, Julien Guihaire, $\mathrm{MD} \mathrm{PhD}{ }^{1,2}$

INSITUTIONAL AFFILIATION:

Adult Cardiac Surgery Department, Marie Lannelongue Hospital, University of South Paris, Le Plessis Robinson, France

Cardiac Pathology Department, Institut Mutualiste Montsouris, 42 Bd Jourdan 75014 Paris, France

CORRESPONDING AUTHOR: Dr Monnier Shraer Nathanael, MD, Institut Mutualiste Montsouris, 42 Bd Jourdan 74014 Paris, France. Email:nshraer@gmail.com. Tel: +33156616783.

DATA AVAILABILITY STATEMENT : The data within this work has been collected in the operative theatre and from patients' records in Marie Lannelongue Hospital.

FUNDING STATEMENT: None

CONFLICT OF INTEREST : None

ETHICS APPROVAL STATEMENT : Use of personal data for clinical research and the design of the study was approved by the Commission National Informatique et Libertes (CNIL n 918230).

INFORMED CONSENT STATEMENT: A letter was sent to all patients for detailed information about the use of personal data for clinical research, and their consent was obtained. 


\section{ABSTRACT}

OBJECTIVES: The supra-annular aortic valve replacement (SA-AVR) allows for implantation of larger prostheses. We describe the technique, early post-operative outcomes and hospital mortality.

METHODS: Patients who underwent SA-AVR with the Carpentier-Edwards Magna Ease bioprosthesis between December 2010 and December 2017 were retrospectively reviewed. The prosthesis was sutured to the aortic annulus along the coronary sinuses, and in a supra-annular position along the non-coronary sinus.

RESULTS: 115 patients were included (mean age: 71,6 years $\pm 9,4$ ). Mean bioprosthesis diameter was 23,3 $\pm 1,7 \mathrm{~mm}$. Four early deaths were observed in the ICU. Early post-operative complications included: reoperation for bleeding $(n=6)$, tamponade $(n=7)$, permanent atrioventricular block $(n=4)$ and hemodialysis $(\mathrm{n}=2)$. In-hospital mortality was $3,48 \%$. Postoperative echocardiography showed a marked decrease in the mean left ventricle - aorta gradient $(50,4 \pm 16,1 \mathrm{mmHg}$ vs $11,3 \pm 4,05 \mathrm{mmHg})$.

CONCLUSION: SA-AVR is safe and associated with favourable immediate outcomes in patients with small aortic annulus.

\section{INTRODUCTION}

Surgical aortic valve replacement (AVR) is recommended for severe aortic valve disease. Bioprosthesis remains the substitute of choice for elderly patients ${ }^{1}$. However, it may be associated with patient-prosthesis mismatch particularly for patients with small aortic annulus ${ }^{2,3}$. Moreover, "valve-in-valve" transcatheter aortic valve replacement (TAVR) has become a suitable therapeutic option for bioprosthesis structural degeneration, especially in patients with high surgical risk ${ }^{4}$. It is therefore of great importance to implant the widest bioprosthesis as possible at the first AVR. We report a surgical technique allowing the implantation of a larger bioprosthesis in patients with small aortic annulus: the supra-annular aortic valve replacement (SAAVR) above the aortic annulus. We analyzed the postoperative outcomes using the Perimount Magna Ease (Carpentier-Edwards, Irvine, California) bioprosthesis on patients who underwent the SA-AVR technique.

\section{SURGICAL TECHNIQUE}

After median sternotomy, cardiopulmonary bypass (CPB) was established between the ascending aorta and the right atrium. The aorta was cross-clamped and a high potassium warm cardioplegia was infused and repeated every 15 minutes. The aortotomy was made above the sinuses and the aortic valve was removed. Combined procedures were performed either before (coronary artery bypass grafting, mitral valve repair or replacement) or after (ascending aorta replacement) the SA-AVR. The size of the annulus was measured with Perimount Magna Ease sizers (Carpentier-Edwards). The prosthesis was chosen one size larger than the maximal sizer that could be inserted, which was also used to determine the appropriate height for insertion of the sutures along the wall of the non-coronary sinus. Four to five pledget stitches were placed on the aortic wall along the non-coronary sinus, at the top of the commissural plane, in U shape with the pledget outside the aorta (Figure 1). The rest of the stitches along the left and right coronary sinuses were placed as usual through the annulus plane. All stitches were equally distributed on the bioprosthesis and knotted (Figure 1). The aortotomy was then carefully closed. De-airing and cardiopulmonary bypass weaning were performed and the patient was safely closed.

\section{COMMENTS}

We were able to evaluate the outcomes of this technique on 115 patients included between December 2010 and December 2017 in our institution. Mean age was $71.6 \pm 9.4$ years (55\% males) and mean BMI was $27.8 \pm 4.54$. Indications for surgery were aortic stenosis $(88.7 \%)$, aortic regurgitation $(6.1 \%)$, combined aortic valve disease $(2.6 \%)$ and infectious endocarditis (2.6\%). Mean aortic valve area was $0.80 \pm 0.26$ $\mathrm{cm}^{2}$ (indexed: $0.44 \pm 0.12 \mathrm{~cm}^{2}$ ) and mean left ventricle $(\mathrm{LV})$ - aortic gradient was $50.4 \pm 16.1 \mathrm{~mm} \mathrm{Hg}$. The implanted bioprosthesis diameter was $23 \mathrm{~mm}$ or more in 89 patients (79.5\%) (mean diameter: $23.3 \pm$ $1.67 \mathrm{~mm}$ ). Additional surgical procedures were combined to SA-AVR in 48 cases (42.8\%), coronary artery bypass grafting being the most frequent. Mean aortic cross clamping time was $74.3 \pm 21.6$ min. There was no 
operative complication associated to the suture of the bioprosthesis directly through the aortic wall of the noncoronary sinus, especially neither aortic dissection nor aortic wall rupture. No patient died during the surgical procedure. Mean intensive care unit (ICU) stay was $3.3 \pm 4.7$ days. Four early deaths $(3.5 \%)$ were observed including 2 multiple organ dysfunctions and 2 septic shocks. Early post-operative complications included: re-operation for bleeding $(n=6)$, tamponade $(n=7)$, severe atrioventricular block requiring permanent pacing $(n=4)$ and hemodialysis for acute renal failure $(n=2)$. No early re-operation for bioprosthesis dysfunction was required. Mean hospitalization stay was $12.8 \pm 5.4$ days. At discharge, mean LV-aortic gradient was 11.3 $\pm 4.1 \mathrm{~mm} \mathrm{Hg}$, mean aortic valve area was $1.87 \pm 0.82 \mathrm{~cm}^{2}$ (indexed: $1.01 \pm 0.45 \mathrm{~cm}^{2}$ ). There were 4 cases of minor periprosthetic leakage $(3.48 \%)$.

Those early outcomes allowed us to conclude that the SA-AVR technique using the Perimount Magna Ease bioprosthesis (Carpentier-Edwards) is safe and associated with favorable hospital outcomes. This technique allows one size larger bioprosthesis to be implanted in patients with small aortic annulus. The incidence of operative complications was low, as well as in-hospital mortality. Short-term echocardiographic results were also unremarkable. The 4 deaths observed in ICU were not related to the bioprosthesis, and there was no higher incidence of atrioventricular block. These findings are consistent with the established results of the Magna Ease bioprosthesis when implanted conventionally ${ }^{5}$. The SA-AVR technique decreases the risk of patient-prosthesis mismatch which remains associated with poor outcomes as recently reported by Fallon et al $^{2}$. However the long-term impact of this non-conventional implantation on the incidence of bioprosthesis degeneration remains to be investigated with a longer follow-up.

ACKNOWLEDGEMENTS AND DISCLOSURES: none

\section{REFERENCES}

1. Pibarot P, Dumesnil JG. Prosthetic heart valves: selection of the optimal prosthesis and long-term management. Circulation. 2009;119:1034-48.

2. Fallon JM, Desimone JP, Brennan JM, et al. The Incidence and Consequence of Prosthesis-Patient Mismatch After Surgical Aortic Valve Replacement. Ann Thorac Surg. 2018;106:14-22.

3. David TE, Uden DE. Aortic valve replacement in adult patients with small aortic annuli. Ann Thorac Surg. 1983;36:577-83.

4. Faerber G, Schleger S, Diab M, et al. Valve-in-valve transcatheter aortic valve implantation: the new playground for prosthesis-patient mismatch. J Interv Cardiol. 2014;27:287-92.

5. Totaro P, Degno N, Zaidi A, Youhana A, Argano V. Carpentier-Edwards PERIMOUNT Magna bioprosthesis: a stented valve with stentless performance? J Thorac Cardiovasc Surg. 2005;130:1668-74.

\section{FIGURE LEGENDS}

Figure 1. Surgical technique of the supra-annular implantation above the aortic annulus using the Perimount Magna Ease aortic bioprosthetic valve. On the left part of the figure, four to five pledget stitches (U shape) are placed along the aortic wall of the non-coronary sinus, 5 to $8 \mathrm{~mm}$ above the aortic annulus. The rest of the stiches are equally distributed at the level of the aortic annulus (left and right coronary sinuses). The final position of the bioprosthesis inside the aortic root is illustrated on the right side of the figure.

Implantation of the Perimount Magna Ease bioprosthetic valve above the aortic annulus 

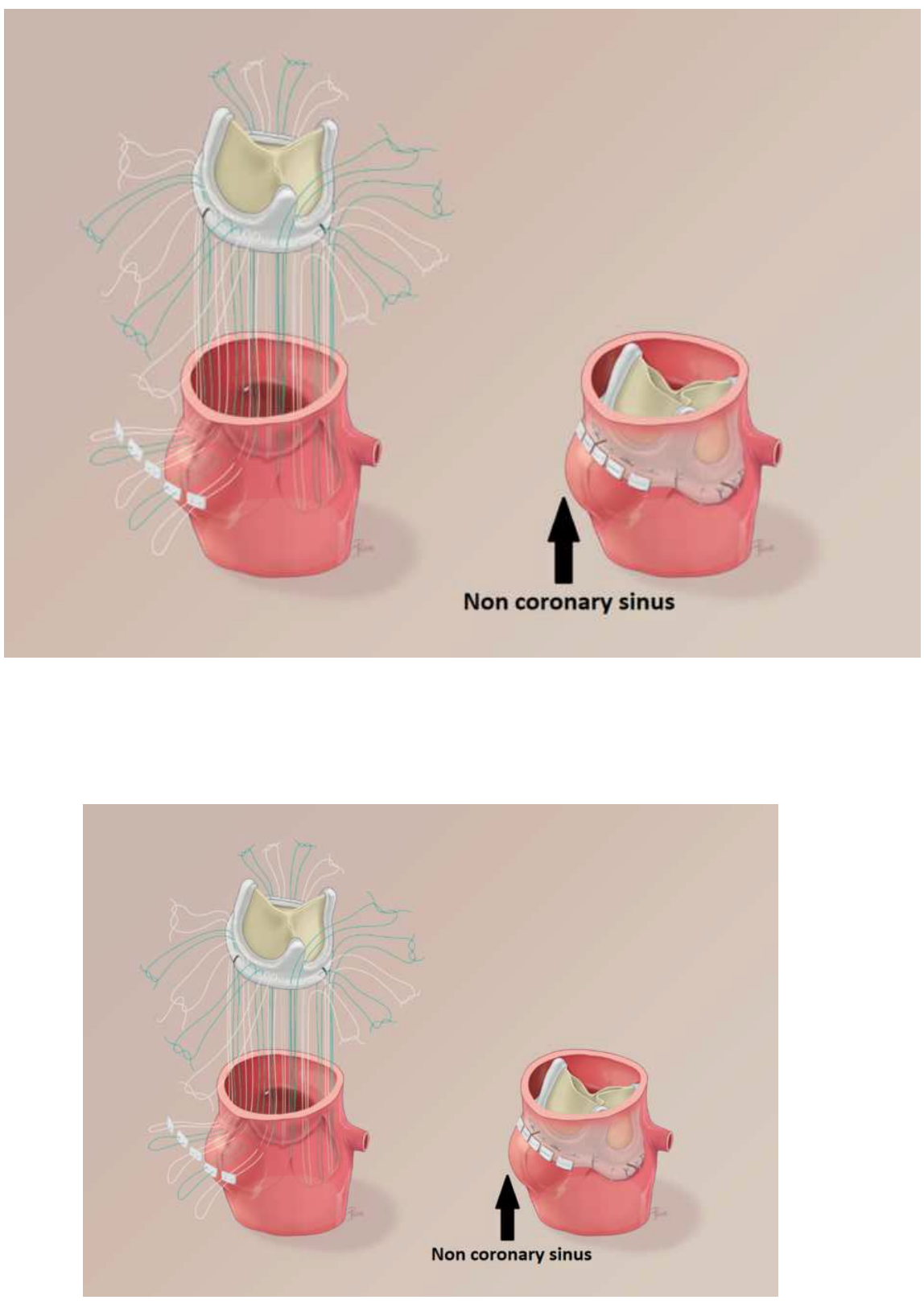